\title{
Evaluation of the Autof MS1000 mass spectrometer in the identification of clinical isolates
}

Qiong Ma', Qi Zhang1', Youhua Yuan', Wenjuan Yan', Shanmei Wang', Junhong Xu', Jiangfeng Zhang ${ }^{1}$, Yuming Wang ${ }^{2^{*}}$ and $\mathrm{Yi} \mathrm{Li}^{1^{*}}$

\begin{abstract}
Background: To evaluate the accuracy and performance of the Autof MS1000 mass spectrometer in bacteria and yeast identification, 2342 isolates were obtained from microbial cultures of clinical specimens (e.g. blood, cerebrospinal fluid, respiratory tract samples, lumbar puncture fluid, wound samples, stool, and urine) collected in 2019 in Henan Provincial People's Hospital. Repetitive strains from the same patient were excluded. We tested the Autof MS1000 and Bruker Biotyper mass spectrometry systems and the classical biochemical identification system VITEK 2/API 20C AUX. Inconsistencies in strain identification among the three systems were identified by 165 rDNA and gene sequencing.
\end{abstract}

Results: At the species level, the Autof MS1000 and Bruker Biotyper systems had isolate identification accuracies of 98.9 and 98.5\%, respectively. At the genus level, the Autof MS1000 and Bruker Biotyper systems were 99.7 and 99.4\% accurate, respectively. The instruments did not significantly differ in identification accuracy at either taxonomic level. The frequencies of unreliable identification were 1.1\% (26/2342) for the Autof MS1000 and 1.5\% (34/2342) for the Bruker Biotyper. In vitro experiments demonstrated that the coincidence rate of the Autof MS1000 mass spectrometer in the identification of five types of bacteria was $>93 \%$, the identification error rate was $<3 \%$, and the no identification rate was 0 . This indicates that the Autof MS1000 system is acceptable for identification.

Conclusions: The Autof MS1000 mass spectrometer can be utilised to identify clinical isolates. However, an upgradation of the database is recommended to correctly identify rare strains.

Keywords: Autof MS1000, Bruker Biotyper, Mass spectrometry, Bacterial identification, Performance verification, Clinical samples

\footnotetext{
* Correspondence: henanyuming@sina.com; liyilabmed@henu.edu.cn

${ }^{2}$ Henan Provincial People's Hospital, Henan University People's Hospital, and People's Hospital of Henan University, Weiwu Road 5, 450003 Zhengzhou, Henan, People's Republic of China

'Department of Clinical Laboratory, Henan Provincial People's Hospital,

Henan University People's Hospital, Zhengzhou 450003, Henan, China
}

(c) The Author(s). 2020 Open Access This article is licensed under a Creative Commons Attribution 4.0 International License, which permits use, sharing, adaptation, distribution and reproduction in any medium or format, as long as you give appropriate credit to the original author(s) and the source, provide a link to the Creative Commons licence, and indicate if changes were made. The images or other third party material in this article are included in the article's Creative Commons licence, unless indicated otherwise in a credit line to the material. If material is not included in the article's Creative Commons licence and your intended use is not permitted by statutory regulation or exceeds the permitted use, you will need to obtain permission directly from the copyright holder. To view a copy of this licence, visit http://creativecommons.org/licenses/by/4.0/ The Creative Commons Public Domain Dedication waiver (http://creativecommons.org/publicdomain/zero/1.0/) applies to the data made available in this article, unless otherwise stated in a credit line to the data. 


\section{Background}

Matrix-assisted laser desorption ionisation-time of flight mass spectrometry (MALDI-TOF MS) is an emerging high-throughput technology with broad potential in clinical microbial identification because of its high resolution, speed, sensitivity, and accuracy [1-3]. Microorganism detection is based on databases of known bacteria. During detection, characteristic protein fingerprints are obtained, and these mass spectra are compared with the database for identification [4-8]. Many companies, such as Bruker Daltonics, bioMérieux, Shimadzu, Beijing Purkinje General Instrument Co., and Autobio Diagnostics, manufacture MALDI-TOF MS instruments. Recently, a new MS, the Autof MS1000 from Autobio Diagnostics, was developed for the identification of clinically important pathogenic bacteria. The Autof MS1000 has some advantages over the existing systems, such as a ion source vacuum (up to $10^{-7} \mathrm{mPa}$ ) and a rapid identification module that delivers a sample result scan in $0.1 \mathrm{~s}$, and can identify an entire target plate (96 isolates) in approximately $21 \mathrm{~min}$. The mass spectrometer has been purchased by many laboratories in China, the United Kingdom, Italy, South Korea, and Thailand. The aim of this study was to evaluate the identification ability of the domestic Autof MS1000 in common clinical microbiology. A commercial Bruker Biotyper mass spectrometer (Bruker Daltonics, Bremen, Germany) was used as the control system. The results provide a reference for further assessment of this instrument in the medical device market.

\section{Results}

Isolate identification

There were no statistically significant differences in the identification of the 2342 strains between the two mass spectrometers at either the species or genus level. The Autof MS1000 and Bruker Biotyper systems had isolate identification accuracies of 98.9 and $98.5 \%$, respectively, at the species level, and 99.7 and $99.4 \%$, respectively, at the genus level. These results demonstrate that the Autof MS1000 and Bruker Biotyper mass spectrometers had equal ability to identify clinical isolates. Detailed results are shown in Fig. 1 and Additional file 1. Common bacteria and yeast were routinely obtained from microbial cultures of clinical specimens, and Escherichia coli, Klebsiella pneumoniae, Acinetobacter baumannii, Staphylococcus aureus, and Pseudomonas aeruginosa were most common clinical isolates. The identification accuracy for common bacteria and yeast reached $98 \%$ on the Autof MS1000. The ability to identify these isolates is of great significance to the evaluation of the MS. Detailed results are shown in Fig. 2 and Additional file 2.

\section{Failure rates}

The Autof MS1000 incorrectly identified or failed to identify $1.1 \%(26 / 2342)$ of the isolates. Of these, 20 strains were identified at the genus level. The Bruker Biotyper incorrectly identified or failed to identify $1.6 \%$ (37/2342) of isolates, 21 of which were accurately identified at the genus level. The two strains of Burkholderia pseudomallei were identified correctly by the Autof MS1000, while the

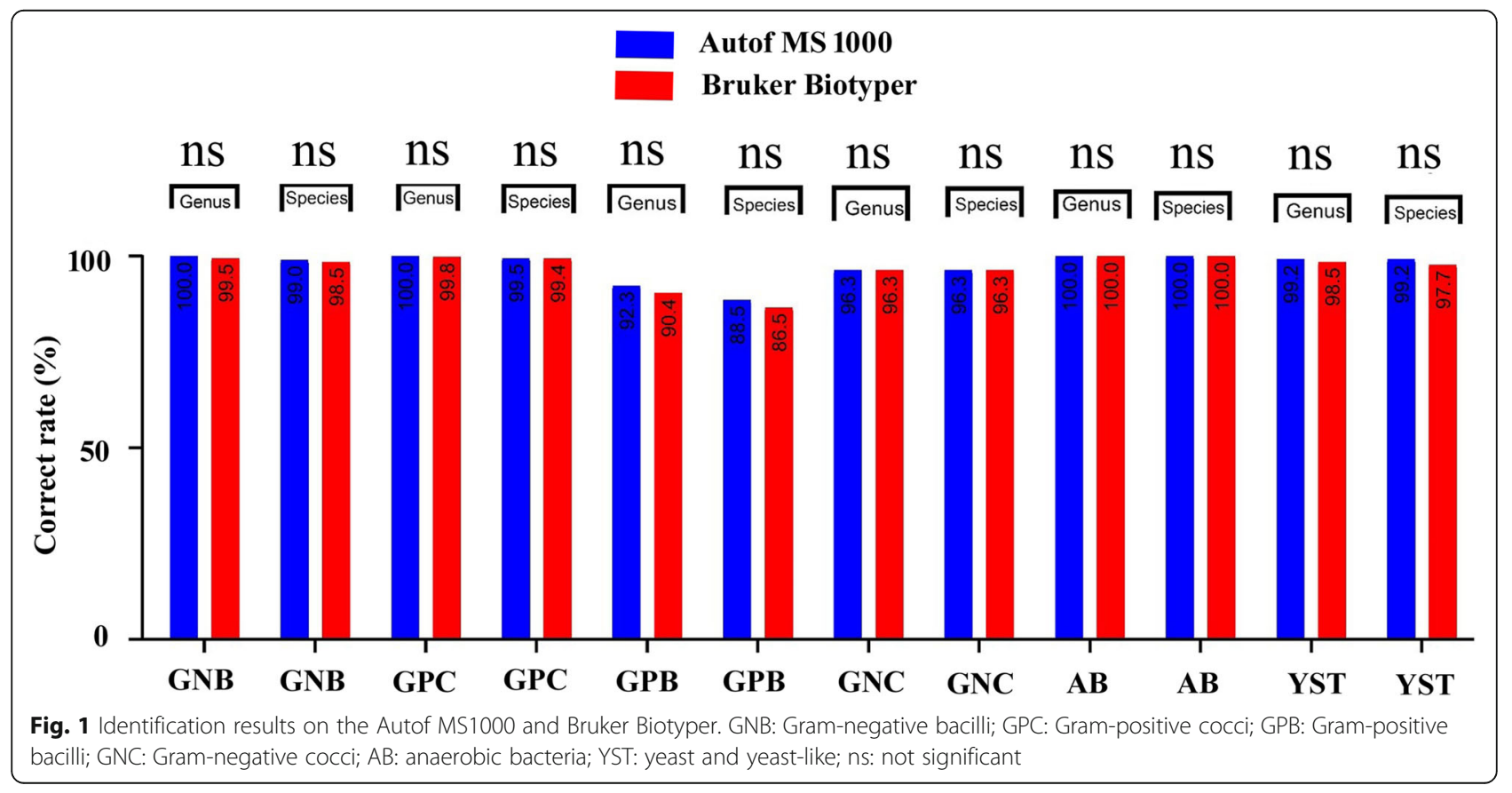




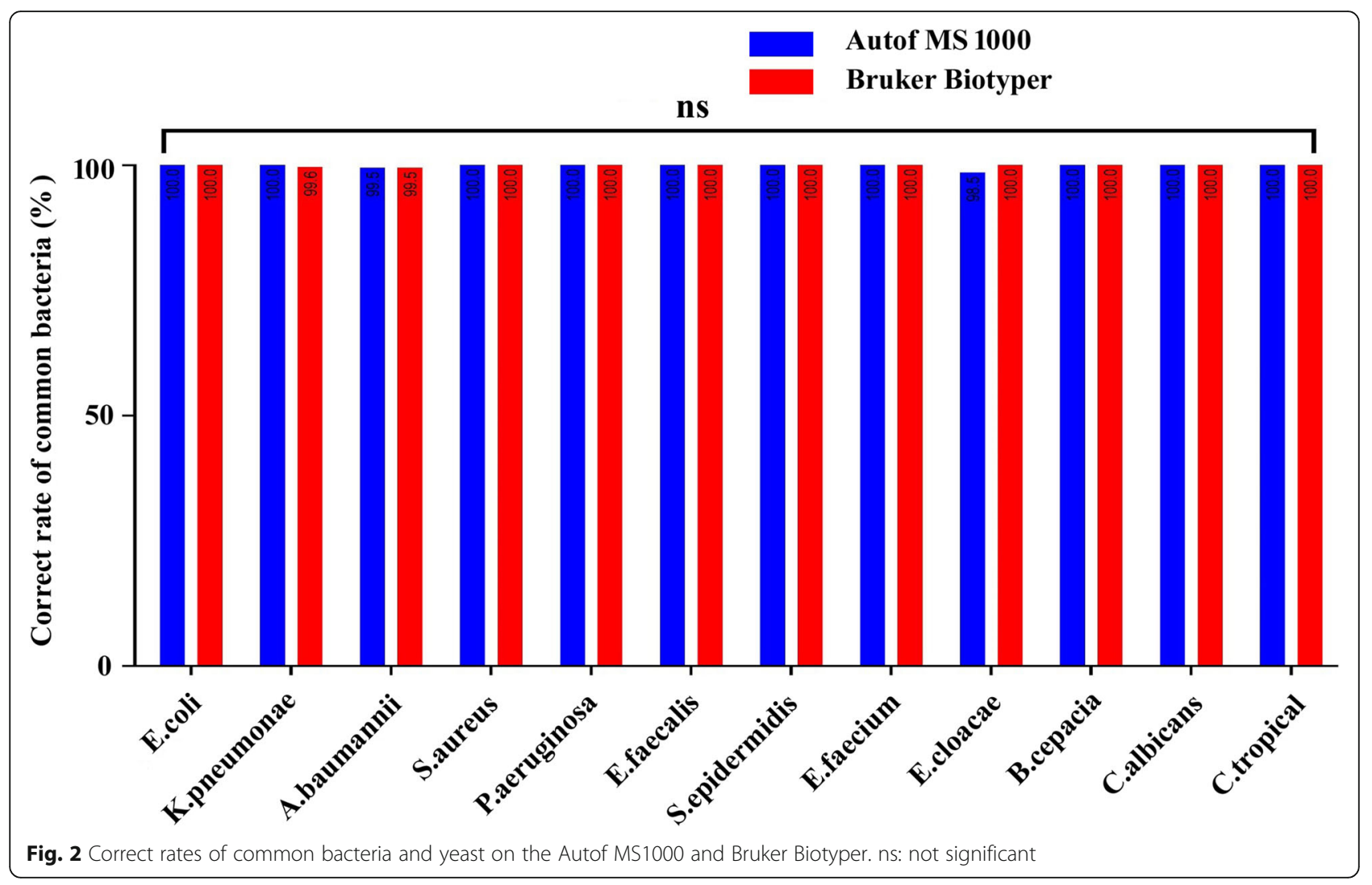

Bruker instrument failed to identify them. B. pseudomallei can cause melioidosis, making it an important strain with clinical significance $[9,10]$. This is a major error and should be noted. The Autof instrument identified nine strains of Salmonella spp. and accurately identified a strain of Salmonella enteritidis to the species level. The Bruker instrument identified eight strains of Salmonella spp. and failed to identify one strain of Salmonella paratyphi A. Neither machine can be used for serotype identification; therefore, Salmonella spp. identified by mass spectrometry will require further serological typing before deciding whether to report an infectious disease. Most other identification errors were minor, such as Citrobacter freundii and Raoultella planticola being erroneously identified as Citrobacter braakii and Raoultella ornithinolytica, respectively (Table 1 ). Fortunately, these results will not affect clinical diagnosis or treatment decisions.

\section{Performance verification}

We evaluated 229 strains of Gram-negative Enterobacteriaceae, Gram-negative non-Enterobacteriaceae, Gramnegative fastidious bacteria, Gram-positive aerobic bacteria, and anaerobic bacteria, as well as yeasts and yeastlike microorganisms, according to the recommendations for the in vitro performance verification of commercial instruments in the Clinical and Laboratory Standards Institute (CLSI) M52 standard [11]. We compared the agreement, discrepancy, and unidentified isolates between the two mass spectrometers. The agreement values of both instruments were $>93 \%$, their discrepancies were $<3$, and $<2 \%$ of isolates were not identified (Fig. 3, Additional file 3). These are all acceptable values, indicating that the Autof MS1000 is a reliable system for isolate identification.

\section{Discussion}

Bacterial identification is of great clinical significance, helping clinicians select antibiotics, accurately treat patients, and improve cure rates. To our knowledge, this is the first assessment of the identification of multiple bacteria using a Chinese mass spectrometer in central China. There were no major differences in the identification of multiple bacteria between the Chinese instrument and an imported mass spectrometer.

MALDI-TOF MS has advanced rapidly in recent years and is gradually replacing biochemical methods as the preferred tool for clinical bacterial identification [1215]. The accuracy of MALDI-TOF MS identification depends on the collection of protein fingerprint data for all possible strains in a database [16]. The Autof MS1000 has a database of 9050 strains and 2727 species, and the Bruker Biotyper database has 5989 strains and 2371 species. Comparing the accuracy of strain identification is primarily a function of comparing strain databases; 
Table 1 Isolates misidentified at the species level or not identified by the Autof MS1000 and Bruker Biotyper

\begin{tabular}{|c|c|c|c|}
\hline 16/18S rRNA identification & $N$ & Autof MS1000 & Bruker Biotyper \\
\hline Acinetobacter baumannii & 1 & Acinetobacter nosocomialis & Acinetobacter nosocomialis \\
\hline Enterobacter cloacae & 1 & Enterobacter cloacae/Enterobacter asburiae & Correct identification \\
\hline Citrobacter freundii & 1 & Citrobacter freundii / Citrobacter braakii & Citrobacter freundii / Citrobacter braakii \\
\hline Aeromonas hydrophila & 1 & Correct identification & Aeromonas hydrophila/Aeromonas caviae \\
\hline Aeromonas hydrophila & 1 & Aeromonas caviae & Aeromonas hydrophila/Aeromonas caviae \\
\hline Salmonella typhimurium & 4 & Salmonella spp. & Salmonella spp. \\
\hline Salmonella enteritidis & 3 & Salmonella spp. & Salmonella spp. \\
\hline Salmonella paratyphi A & 1 & Salmonella enterica & No identification \\
\hline Salmonella enteritidis & 1 & Correct identification & Salmonella spp. \\
\hline Burkholderia pseudomallei & 2 & Correct identification & No identification \\
\hline Raoultella planticola & 1 & Raoultella ornithinolytica & Raoultella ornithinolytica \\
\hline Raoultella planticola & 1 & Raoultella ornithinolytica & Correct identification \\
\hline Aeromonas caviae & 1 & Aeromonas hydrophila / Aeromonas caviae & Correct identification \\
\hline Enterobacter cancerogenus & 1 & Correct identification & Enterobacter cloacae/Enterobacter cancerogenus \\
\hline Leifsonia shinshuensis & 1 & Correct identification & Leifsonia spp. \\
\hline Dysgonomonas gadei & 1 & Correct identification & No identification \\
\hline Staphylococcus hominis & 1 & Correct identification & Staphylococcus haemolyticus \\
\hline Staphylococcus haemolyticus & 1 & Correct identification & Staphylococcus epidermidis \\
\hline Staphylococcus haemolyticus & 1 & Staphylococcus hominis & Staphylococcus hominis \\
\hline Nocardia asteroides & 1 & Correct identification & No identification \\
\hline Nocardia otitidiscaviarum & 1 & No identification & No identification \\
\hline Nocardia brasiliensis & 1 & Nocardia spp. & Nocardia spp. \\
\hline Streptococcus constellatus & 2 & Streptococcus constellatus/Streptococcus anginosus & Correct identification \\
\hline Staphylococcus gallinarum & 1 & Correct identification & No identification \\
\hline Mycobacterium farcinogenes & 1 & Correct identification & No identification \\
\hline Mycobacterium abscessus & 1 & No identification & Correct identification \\
\hline Mycobacterium smegmatis & 1 & No identification & No identification \\
\hline Actinomyces neuii & 1 & Correct identification & No identification \\
\hline Streptomyces violaceoruber & 1 & No identification & Correct identification \\
\hline Bacillus pumilus & 1 & Bacillus altitudinis & Bacillus altitudinis \\
\hline Moraxella catarrhalis & 1 & No identification & No identification \\
\hline Candida glabrata & 1 & Correct identification & Candida spp. \\
\hline Candida rugosa & 1 & No identification & No identification \\
\hline Kazachstania servazzii & 1 & Correct identification & No identification \\
\hline
\end{tabular}

therefore, construction of the database (coverage, type, etc.) is critical [17].

For Gram-negative bacilli isolates requiring species-level identification $(n=1449)$, the analytical accuracies of the two systems were similar (99.0 and $98.7 \%$ for the Autof MS1000 and Bruker Biotyper systems, respectively ( $p=$ $0.490)$ ). However, certain closely related microorganisms cannot be distinguished from one another using MALDITOF MS, such as Aeromonas, Raoultella, Enterobacter, Acinetobacter, and Citrobacter spp. Similar conclusions have been reported by several other researchers, who could not distinguish these closely related species [18-22]. Hence, for closely related species or subspecies, MALDITOF MS should be used in combination with biochemical and molecular methods. For Salmonella spp. identification, the limitations of MALDI-TOF MS must be considered [23]. Biochemical and serological tests will still be required to accurately identify Salmonella spp.

In this experiment, a coagulase-negative staphylococcus was isolated from blood cultures. Species-level reporting is sometimes essential to determine the clinical significance of culture isolates of coagulase-negative 


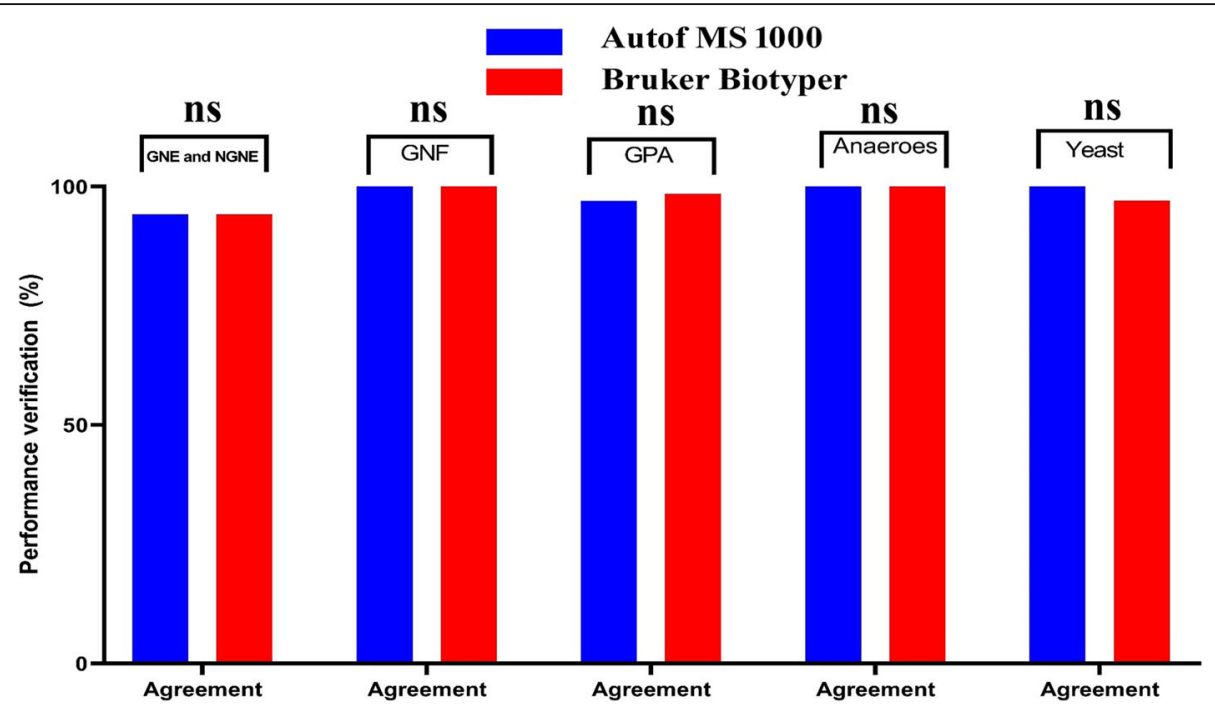

Fig. 3 Performance verification for the Autof MS1000 and Bruker Biotyper. GNE: Gram-negative Enterobacteriaceae; NGNE: Gram-negative nonEnterobacteriaceae; GNF: Gram-negative fastidious; GPA: Gram-positive aerobic; ns: not significant

staphylococci [24]. The Autof MS1000 allowed better identification of Staphylococcus hominis and Staphylococcus haemolyticus than the Bruker Biotyper, suggesting that the Autof MS1000 has increased specificity for the identification of these species. However, this will require further verification with increased sample size and additional species. Viridans streptococci isolates were all correctly identified by the Bruker Biotyper, whereas two strains of Streptococcus constellatus were not identified to the species level by the Autof MS1000. Using the Bruker Biotyper, one strain of Leifsonia shinshuensis, one strain of Staphylococcus gallinarum, two strains of Burkholderia pseudomallei, and one strain of Kazachstania servazzii could not be identified, as these strains were not included in the Bruker Biotyper database (v5.0 5898). Database updates may resolve the difficulties in distinguishing these species.

Among the rare strains that were misidentified, $M y c o-$ bacterium spp., Nocardia spp., and Actinomyces spp. were not correctly identified by either system. MALDI-TOF MS does have limitations in the identification of mycobacteria, Nocardia spp. and other aerobic actinomycetes found in the clinical microbiology laboratory [25]. This is due to the presence of multiple strains, which are not fully represented in the database. Although some strains are included, they cannot be accurately identified even with repeated operations. It may be that the protein profiles they produce are inconsistent with the characteristic profile in the database. In that case, the strain diversity of the database should be increased. Another limitation in the use of MALDI-TOF MS with slowly growing Mycobacterium spp. and Actinomyces spp. is the potential occurrence of the two species in mixed cultures, which will be recognised as the colonies on the culture plate mature, but are misidentified by MALDI-TOF MS. In addition, the sample preparation method may be an important factor for successful identification, particularly for species that are difficult to lyse, such as Mycobacterium spp. and Nocardia spp. A two-step cell disruption protocol combining the use of $0.5-\mathrm{mm}$ diameter silica/zirconia beads and sonication for $15 \mathrm{~min}$ greatly improves the efficacy of mycobacterial identification by MALDI-TOF MS [26].

This study has some limitations. First, the sample size should be increased, and the species detected should be expanded to include more rare bacteria. Second, we did not evaluate the identification of filamentous fungi. Therefore, we will increase the sample size and analyse filamentous fungi identification in subsequent evaluations.

\section{Conclusions}

In summary, both the Autof MS1000 and Bruker Biotyper meet the clinical requirements for bacterial identification. However, for some closely related bacteria, accurate identifications should be obtained by combining morphological, phenotypic, and molecular characteristics. A lack of diversity in database strains is also a major factor affecting the ability to identify bacteria by MALDI-TOF MS [27]. MALDI-TOF MS databases are constantly expanding, and instrument databases should be regularly updated to ensure optimal isolate identification.

\section{Methods}

Sample collection

A total of 2342 clinical isolates, excluding duplicate strains (172 species, 76 genera) were obtained from 
bacterial cultures of clinical specimens (e.g. blood, cerebrospinal fluid, respiratory tract samples, lumbar puncture fluid, wound samples, pus, ear secretion, stool, and urine) collected at Henan Provincial People's Hospital (Zhengzhou, China) in 2019. This study was approved by the Ethics Committee of Henan Provincial People's Hospital, Henan, China (20190050). The 2342 clinical isolates contained aerobic Gram-negative bacilli (1449 strains), aerobic Gram-negative cocci (27 strains), aerobic Gram-positive bacilli (52 strains), aerobic Grampositive cocci (659 strains), anaerobes (48 strains), and yeasts (108 strains). Fresh samples were cultured using a variety of commonly used solid media, including tryptic soy agar with $5 \%$ sheep's blood (BAP), chocolate agar (CHOC), and Sabouraud dextrose agar (SAB). Most specimens were incubated for $18-24 \mathrm{~h}$ at $36 \pm 1{ }^{\circ} \mathrm{C}$, whereas some required additional time for sufficient growth. For example, some anaerobes required up to 72 $\mathrm{h}$ of incubation for reliable species-level identification.

\section{Quality control}

E. coli (American Type Culture Collection (ATCC)25,922), S. aureus (ATCC29213), P. aeruginosa (ATCC27853), Enterococcus faecalis (ATCC51299), Enterococcus faecium (ATCC19434), Bacteroides fragilis (ATCC25285), and Candida albicans (ATCC10231) were used as reference strains. A microorganism identification calibrator was used for the Autof MS1000 and an IVD BTS solution calibrator was used for the Bruker Biotyper. Negative controls consisted of reagents only (usually $\alpha$-cyano-4-hydroxycinnamic acid matrix) and were included to detect false-positive results and reagent contamination. The Bruker Biotyper uses non-disposable target slides, and the negative control was placed at different target positions in different runs to control for locationbased differences. The Autof MS1000 uses disposable target slides; therefore, the negative control was not used.

\section{Instruments and reagents}

A Bruker Biotyper system (Bruker Daltonics, Bremen, Germany), an Autof MS1000 system (Autobio Diagnostics, Zhengzhou, China), and supporting consumables from the respective manufacturers were used. Reference strains were obtained from the ATCC (Manassas, VA, USA). Other materials, including BAP, CHOC, and SAB, were purchased from Zhengzhou Autobio Co., Ltd. (Zhengzhou, China).

\section{Identification using the Vitek 2 compact and API 20C AUX system}

Based on colony morphology and staining results, a corresponding identification card was selected for each isolate. Identification results were automatically interpreted by the system according to the product manual, using the established algorithm. When the isolate was properly assigned to a given species or identified with low discrimination but resolved by supplemental tests, the identification was considered reliable.

\section{Bacterial identification by MALDI-TOF MS}

MS quality control and operation were performed according to the CLSI M58 standard [28] and the Chinese Expert Consensus for Clinical Microbial Mass Spectrometry Application [29]. Deposit preparation and analysis were similar for both systems. For the Autof MS1000, protein spectra were analysed with Autof Acquirer version 1.0.55 software and library v1.1.09050. The manufacturer's interpretation criteria were applied, with identification scores $\geq 9$ considered positive at the species level, scores of 6-9 considered positive at the genus level, and scores $<6$ defined as not identified.

On the Bruker Biotyper, extraction procedures were performed according to the product manual. Protein spectra were analysed with Bruker Biotyper 3.1 software and library v5.0 5898. The manufacturer's interpretation criteria were applied, with identification scores $\geq 2.0$ considered positive at the species level, scores of 1.7-2 considered positive at the genus level, and scores $<1.7$ defined as not identified.

\section{Sequencing}

For certain strains, when both mass spectrometry identifications and the biochemical identification were inconsistent at the species level, the isolate was sent to Beijing Ruiboxing Co., Ltd. (Beijing, China) for confirmation by sequencing. If the mass spectrometry identifications at the species and genus levels were inconsistent with the results of $16 \mathrm{~S}$ rDNA sequencing, then the mass spectrometry results were considered incorrect. The $16 \mathrm{~S}$ rRNA genes of all bacteria were sequenced, along with $d n a J$, $\operatorname{sod} A$, tuf, or ropB for Gram-positive cocci [30, 31]; rop $B$, gyrB, recA, or cpn60 for Gram-negative bacteria [30, 32]; and $\operatorname{ropB}, \operatorname{gyr} B$, SecA1, or hsp65 for Grampositive bacilli [30, 33]. For yeasts, the internal transcribed spacer located between the nuclear $18 \mathrm{~S}$ and $26 \mathrm{~S}$ rRNA genes was sequenced [30].

\section{Selection principles for the performance evaluation strains}

Non-reference methods were used for comparison according to the CLSI M52 standard for the verification of the in vitro performance of commercial instruments [11]. Five kinds of bacteria (Gram-negative Enterobacteriaceae, Gram-negative non-Enterobacteriaceae, Gramnegative fastidious bacteria, Gram-positive aerobic bacteria, and anaerobic bacteria), and yeast-like fungi were evaluated using three parameters: the agreement (\% agreement), identification error (\% discrepancy) and unidentified species (\% not identified) rates. Methods with 
$\geq 93 \%$ agreement, $<3 \%$ discrepancy, and $<2 \%$ of species not identified were considered acceptable.

\section{Statistical analysis}

Statistical analysis was performed using SPSS 20.0 statistical analysis software (IBM Corporation, Armonk, NY, $U S A)$. Categorical variables were compared with Chisquared or Fisher's exact tests. Two-tailed $p$ values < 0.05 were considered statistically significant. Figures were generated using GraphPad Prism version 8.0 (GraphPad Software Inc., La Jolla, CA, USA).

\section{Supplementary information}

Supplementary information accompanies this paper at https://doi.org/10. 1186/s12866-020-02005-0.

Additional file 1. Identification results on the Autof MS1000 and Bruker Biotyper

Additional file 2. MS analysis of common bacteria and yeasts on the Autof MS1000 and Bruker Biotyper

Additional file 3. Verification of microbial identification on the Autof MS1000 and Bruker Biotyper

\section{Abbreviations}

MALDI-TOF MS: Matrix-assisted laser desorption ionisation-time of flight mass spectrometry; CLSI: Clinical and Laboratory Standards Institute; ATCC: American Type Culture Collection; BAP: Tryptic soy agar with 5\% sheep's blood; CHOC: Chocolate agar; SAB: Sabouraud dextrose agar

\section{Acknowledgements}

Not applicable

\section{Authors' contributions}

$\mathrm{QM}, \mathrm{YY}$, and $\mathrm{YL}$ contributed to the writing of the manuscript; $\mathrm{SL}, \mathrm{SW}$, and JX analysed and interpreted the experimental data; WY, JZ, QZ, and MG analysed the mass spectrometry results; $Y Y$ and QM performed statistical analyses and data plotting. The authors read and approved the final manuscript.

\section{Funding}

This study was supported by the Joint Programs of the Henan Provincial Government and Healthy Ministry (SB2018084, SB20190318, and LHGJ20190611) and Henan Provincial Key Programs in Science and Technology (181200211900 and 202102310355). The funders had no role in the design of the study, the collection, analysis, and interpretation of data, or in the writing the manuscript.

\section{Availability of data and materials}

The data sets used during the current study are available from the corresponding author on reasonable request.

\section{Ethics approval and consent to participate}

This study was approved by the Ethics Committee of Henan Provincial People's Hospital, Henan, China (20190050); the need for written informed consent was waived because de-identified retrospective data were used. The permission of the corresponding author was required to access the raw data/samples.

\section{Consent for publication}

Not applicable.

\section{Competing interests}

The authors declare that they have no competing interests.
Received: 19 May 2020 Accepted: 12 October 2020

Published online: 20 October 2020

\section{References}

1. Singhal N, Kumar M, Kanaujia PK, Virdi JS. MALDI-TOF mass spectrometry: an emerging technology for microbial identification and diagnosis. Front Microbiol. 2015:6:791.

2. Jang KS, Kim YH. Rapid and robust MALDI-TOF MS techniques for microbial identification: a brief overview of their diverse applications. J Microbiol. 2018;56:209-16

3. Martiny D, Busson L, Wybo I, El Haj RA, Dediste A, Vandenberg O. Comparison of the microflex LT and Vitek MS systems for the routine identification of bacteria by matrix-assisted laser desorption-ionization timeof-flight mass spectrometry. J Clin Microbiol. 2012;50:1313-25.

4. Pan C, Xu S, Zhou H, Fu Y, Ye M, Zou H. Recent developments in methods and technology for analysis of biological samples by MALDI-TOF-MS. Anal Bioanal Chem. 2007;387:193-204.

5. Egelhofer $\mathrm{V}$, Büssow K, Luebbert C, Lehrach H, Nordhoff E. Improvements in protein identification by MALDI-TOF-MS peptide mapping. Anal Chem. 2000;72:2741-50.

6. Egelhofer V, Gobom J, Seitz H, Giavalisco P, Lehrach H, Nordhoff E. Protein identification by MALDI-TOF-MS peptide mapping: a new strategy. Anal Chem. 2002;74:1760-71.

7. $\quad$ Meng Q, Ge S, Yan W, Li R, Dou J, Wang H, et al. Screening for potential serum-based proteomic biomarkers for human type 2 diabetes mellitus using MALDI-TOF MS. Proteomics Clin Appl. 2017;11:1600079.

8. Wang $Y$, Chen XF, Xie XL, Xiao M, Yang Y, Zhang G, et al. Evaluation of VITEK MS, Clin-ToF-II MS, autof MS1000 and VITEK 2 ANC card for identification of Bacteroides fragilis group isolates and antimicrobial susceptibilities of these isolates in a Chinese university hospital. J Microbiol Immunol Infect. 2019;52:456-64.

9. Yuan Y, Yao Z, Xiao E, Zhang J, Wang B, Ma B, et al. The first imported case of melioidosis in a patient in Central China. Emerg Microbes Infect. 2019;8: 1223-8.

10. Harch SAJ, Currie BJ, Papanicolas L, Rigas V, Baird R, Bastian I. Utility of a rapid lateral flow assay to resolve erroneous identification of Burkholderia pseudomallei as Burkholderia thailandensis by MALDI-TOF mass spectrometry. J Clin Microbiol. 2018;56:e01437-18.

11. Clinical and Laboratory Standards Institute. Verification of commercial microbial identification and antimicrobial susceptibility testing systems. 1st ed. Wayne: Clinical and Laboratory Standards Institute; 2015.

12. Dingle TC, Butler-Wu SM. MALDI-TOF mass spectrometry for microorganism identification. Clin Lab Med. 2013;33:589-609.

13. Park JS, Choi SH, Hwang SM, Hong YJ, Kim TS, Park KU, et al. The impact of protein extraction protocols on the performance of currently available MALDI-TOF mass spectrometry for identification of mycobacterial clinical isolates cultured in liquid media. Clin Chim Acta. 2016;460:190-5.

14. Ceballos-Garzón A, Cortes G, Morio F, Zamora-Cruz EL, Linares MY, Ariza BE, et al. Comparison between MALDI-TOF MS and MicroScan in the identification of emerging and multidrug resistant yeasts in a fourth-level hospital in Bogotá, Colombia. BMC Microbiol. 2019;19:106.

15. Dec M, Puchalski A, Urban-Chmiel R, Wernicki A. 16S-ARDRA and MALDlTOF mass spectrometry as tools for identification of Lactobacillus bacteria isolated from poultry. BMC Microbiol. 2016;16:105.

16. Alatoom AA, Cunningham SA, Ihde SM, Mandrekar J, Patel R. Comparison of direct colony method versus extraction method for identification of grampositive cocci by use of Bruker Biotyper matrix-assisted laser desorption ionization-time of flight mass spectrometry. J Clin Microbiol. 2011:49:2868-73.

17. Lévesque S, Dufresne PJ, Soualhine H, Domingo M-C, Bekal S, Lefebvre B, et al. A side by side comparison of Bruker Biotyper and VITEK MS: utility of MALDI-TOF MS technology for microorganism identification in a public health reference laboratory. PLoS One. 2015;10:e144878.

18. Vávrová A, Balážová T, Sedláček I, Tvrzová L, Šedo O. Evaluation of the MALDI-TOF MS profiling for identification of newly described Aeromonas spp. Folia Microbiol (Praha). 2015:60:375-83.

19. Sekowska A, Mikucka A, Gospodarek-Komkowska E. Identification of Raoultella spp.: comparison of three methods. Indian J Med Microbiol. 2018; 36:197-200

20. Böhme K, Fernández-No IC, Barros-Velázquez J, Gallardo JM, Calo-Mata P, Cañas B. Species differentiation of seafood spoilage and pathogenic gram- 
negative bacteria by MALDI-TOF mass fingerprinting. J Proteome Res. 2010; 9:3169-83.

21. Šedo O, Radolfová-KY̌ižová L, Nemec A, Zdráhal Z. Limitations of routine MALDI-TOF mass spectrometric identification of Acinetobacter species and remedial actions. J Microbiol Methods. 2018;154:79-85.

22. Książczyk M, Kuczkowski M, Dudek B, Korzekwa K, Tobiasz A, KorzeniowskaKowal A, et al. Application of routine diagnostic procedure, VITEK 2 compact, MALDI-TOF MS, and PCR assays in identification procedure of bacterial strain with ambiguous phenotype. Curr Microbiol. 2016;72:570-82.

23. Deng J, Fu L, Wang R, Yu N, Ding $X$, Jiang L, et al. Comparison of MALDITOF MS, gene sequencing and the Vitek 2 for identification of seventy-three clinical isolates of enteropathogens. J Thorac Dis. 2014;6:539-44.

24. Argemi X, Riegel P, Lavigne T, Lefebvre N, Grandpré N, Hansmann Y, et al. Implementation of matrix-assisted laser desorption ionization-time of flight mass spectrometry in routine clinical laboratories improves identification of coagulase-negative staphylococci and reveals the pathogenic role of Staphylococcus lugdunensis. J Clin Microbiol. 2015;53:2030-6.

25. Buckwalter SP, Olson SL, Connelly BJ, Lucas BC, Rodning AA, Walchak RC, et al. Evaluation of matrix-assisted laser desorption ionization-time of flight mass spectrometry for identification of Mycobacterium species, Nocardia species, and other aerobic Actinomycetes. J Clin Microbiol. 2016;54:376-84.

26. O'Connor JA, Lynch-Healy M, Corcoran D, O'Reilly B, O'Mahony J, Lucey B. Improved matrix-assisted laser desorption ionization-time of flight mass spectrometry (MALDI-TOF MS)-based identification of Mycobacterium spp. by use of a novel two-step cell disruption preparatory technique. J Clin Microbiol. 2016:54:495-6.

27. Vlek A, Kolecka A, Khayhan K, Theelen B, Groenewald M, Boel E, et al. Interlaboratory comparison of sample preparation methods, database expansions, and cutoff values for identification of yeasts by matrix-assisted laser desorption ionization-time of flight mass spectrometry using a yeast test panel. J Clin Microbiol. 2014;52:3023-9.

28. Clinical and Laboratory Standards Institute. Methods for the identification of cultured microorganisms using matrix-assisted laser desorption/ionization time-of-flight mass spectrometry. 1st ed. Wayne: Clinical and Laboratory Standards Institute; 2017

29. Expert group on consensus of clinical microbiology mass spectrometry in China. Chinese expert consensus for clinical microbial mass spectrometry application. Chin J Nosocomial. 2016;26:2149-52.

30. Clinical and Laboratory Standards Institute. Interpretive criteria for identification of bacteria and fungi by DNA target sequencing. 2nd ed. Wayne: Clinical and Laboratory Standards Institute; 2018.

31. Bergeron M, Dauwalder O, Gouy M, Freydiere A-M, Bes M, Meugnier H, et al. Species identification of staphylococci by amplification and sequencing of the tuf gene compared to the gap gene and by matrix-assisted laser desorption ionization time-of-flight mass spectrometry. Eur J Clin Microbiol Infect Dis. 2011:30:343-54.

32. Miñana-Galbis D, Urbizu-Serrano A, Farfán M, Fusté MC, Lorén JG. Phylogenetic analysis and identification of Aeromonas species based on sequencing of the cpn60 universal target. Int J Syst Evol Microbiol. 2009;59: 1976-83.

33. Maleki MR, Kafil HS, Harzandi N, Moaddab SR. Identification of nontuberculous mycobacteria isolated from hospital water by sequence analysis of the hsp65 and 165 rRNA genes. J Water Health. 2017;15:766-74.

\section{Publisher's Note}

Springer Nature remains neutral with regard to jurisdictional claims in published maps and institutional affiliations.

Ready to submit your research? Choose BMC and benefit from:
- fast, convenient online submission
- thorough peer review by experienced researchers in your field
- rapid publication on acceptance
- support for research data, including large and complex data types
- gold Open Access which fosters wider collaboration and increased citations
- maximum visibility for your research: over 100M website views per year
At BMC, research is always in progress.
Learn more biomedcentral.com/submissions

